\title{
Numerical Modelling and Experimental Validation of Twin-Screw Pumps Based on Computational Fluid Dynamics using SCORG ${ }^{\circledR}$ and SIMERICS MP+ ${ }^{\circledR}$
}

\author{
Pasquale Borriello ${ }^{1, *}$, Emma Frosina ${ }^{2}$, Adolfo Senatore $^{1}$, Federico Monterosso $^{3}$ \\ ${ }^{1}$ University of Naples Federico II, Department of Industrial Engineering, Via Claudio, 21 - 80125 \\ Naples, Italy \\ ${ }^{2}$ University of Sannio, Department of Engineering, Piazza Roma, 21 - 82100 Benevento, Italy \\ ${ }^{3}$ Omiq s.r.l., Via Serviliano Lattuada, 31 - 20135 Milano, Italy
}

\begin{abstract}
This paper presents a methodology for simulating screw pumps using a 3D-CFD transient approach. It is known in literature that the advantages of screw pumps in noise emission, reliability, and their capacity to work with any kind of fluid make their applications interesting for many fields. Increasing demands for high-performance screw pumps require a deep understanding of the flow field inside the machine. The investigation is performed by use of a 3D computational fluid dynamics analysis based on a single-domain structured moving mesh obtained by novel grid generation procedure through the commercial software SCORG. The real-time mass flow rate, rotor torque, pressure distribution, velocity field, and other performance indicators including the indicated power were obtained from numerical simulations performed in the SimericsMP+ environment. The performance curves of the numerical model were produced for variable rotation speeds and discharge pressures and compared with experimental data with high accuracy. The pressure distribution in the screw groove is relatively uniform, the screw clearance and the meshing area pressure are different from the screw groove pressure distribution. The results demonstrate that the speed does not have a considerable effect on the pressure field. At last, the numerical model was validated by comparing the numerical results with the measured performance obtained in the experimental test rig through the comprehensive experiment performed for a set of discharge pressures and rotational speeds. The model has shown to predict pressure variation and flow rate with good accuracy.
\end{abstract}

\section{Introduction}

Screw pumps have the characteristics of being simple and having small volume, high ability of self-priming, smooth working, long life span, easy disassembly, low vibration, no pressure fluctuation and noise; so, they are widely used in petrochemical, shipping, energy and food industries. Increasing demands for high-performance screw pumps require improvements in pump designs. Recent developments in manufacturing technologies allow accurate production of novel designs. On the other hand, the spindle kinematics and internal flow field are very complex, so that the number of available flow studies on screw pumps is quite limited, and the understanding of the internal flow is yet incomplete. Therefore, for

* Corresponding author: pasquale.borriello@unina.it 
improvements in the design of screw pumps, the full understand of the process within the pump is required. To date, most of the models for their performance analysis are based on thermodynamic chamber modelling. Research works on modelling and experimental investigation of working processes in twin-screw pumps was reported in many previous studies. The number of literature resources is large and therefore only the most relevant have been shown in this paper. Li [1] and [2] reviewed the compositions of the screw pump, various types of structures and the calculation of the generation and the performance of the tooth profile. Moreover, they discussed systematically the structure, rotor profiles generation and performance calculation of different screw pump profiles and optimized some of the profiles to achieve better sealing. Vetter and Wincek [3, 4] developed the first analytical model to predict the volumetric flow capacity for both single-phase and two-phase operation. Mewes et al. [5] proposed a performance calculation model for a multiphase pump according to the mass and energy conservation in the pump chamber and validated it by experiments. Patil [6] and Chan [7] studied the steady state and transient properties under different working conditions. They discussed about the influence of the viscosity of the sealing liquid and gas void fraction on the performance of a two-phase screw pump. Rabiger [8] proposed a model for a screw pump and carried out a numerical and experimental analysis of the performance of the pump under very high gas volume fractions (90-99\%). Rabiger also conducted an experiment to visualize the leakage flow in radial clearances [9]. Cao et al. [10] proposed calculation models for the back flow and pressure distribution within the multiphase twinscrew pump and simulated the thermodynamic performance of the pump with different gas volume fractions. Hu et al. [11] established a theoretical model to evaluate the twin-screw pump clearance, the total pump volumetric flow, and the return flow in the power consumption for various pressure differentials and gas clearance fractions. Model predictions were found in good agreement with the experimental data. Liu et al. [12] developed an analytical model to predict the multi-phase performance of the twin-screw pump and to simulate the leakage flow in the clearance, which showed that the analytical model prediction and the experimental data were well matched.

The literature resources referenced above provide a good understanding of the working process but point out that improvements are possible especially for novel applications of multiphase pumps. However, most of the current methods are based on the thermodynamic chamber mathematical models which neglect kinetic energy and simplify the analysis of the main and leakage flows. Few others refer to a steady state CFD which assumes static mesh of the moving flow domains. Indeed, R. Ma and K. Wang [13] modelled the quasi-steady flow in the pump with different clearances in multiple reference frame (MRF) of CFD. The pump characteristics experiments are also carried out. Tang and Zhang [14, 15, 16] established a CFD numerical model with a static grid, proposed a leak model for the twinscrew pump, and optimized the tooth profile: a new solving transcendental equation method combining graphics and analysing approach-Matlab Anonymous Function Method (MAFM) was developed to solve the screw contact line equation.

By using static mesh, approximate pressure gradients and the leakage velocity field can be obtained; however, such results do not take into account the velocity field of the main flow and neglect the transient nature of the working process in a screw pump. Due to the limitation of CFD simulation using static mesh, some important parameters cannot be obtained, such as the mass flow rate, rotor torque and pressure fluctuation. Therefore, any dynamic behaviour including cavitation and multiphase flows could not be fully analysed using such static mesh.

The working domain of a screw pump is formed between moving rotors and stationary casing. It changes the shape and size with the rotation of the rotors. To obtain the pressure, temperature and velocity fields in CFD simulations, the numerical mesh needs to deform in time accurately following the shape of the working domain. The numerical grid generators 
commonly implemented in the commercial CFD software are unable to fulfil these requirements and a specialized grid generator is required. Breakthrough in using CFD for analysis of positive displacement screw machines was made by Kovacevic et al. [17] who generated a structured moving mesh for screw compressor rotor based on a rack generation method proposed by Stosic et al. [18]. This pioneering work in grid generation for screw machines allowed for the CFD simulations and performance prediction of screw compressors. This method provided a powerful basis for research of screw pumps. Yan et al. [19] built up a full 3D CFD model of twin-screw pump using structured moving numerical mesh with the conformal interface between the rotor domains based on FVM. The calculation results were validated with experimental data, to confirm the accuracy and applicability of this numerical method for performance prediction of screw pumps. The same authors [20] also analysed difference in performance of 2-3 lobe combination of twin-screw pumps with different rotor profiles, known as A-type, B-type, C-type and D-type, by means of CFD and $\mathrm{SCORG}^{\circledR}$ for meshing the screws. The comparison of results obtained with two rotor profiles gave an insight on the advantages and disadvantages of each of them. Hai-Tao YU1 [21] and Di Zhang et al [22] focused on the research and development of the twin-screw pumps, including a screw rotor profile design, the hydraulic characteristics of the twin-screw pump and the effects of the speed and the screw clearance on the performance of the pump based on CFD using Pumplinx ${ }^{\circledR}$ and SCORG $^{\circledR}$ as mesh generator. Zhang et al. [23] compared experimental data and URANS CFD results to check the CFD closure models' validity focusing on leakage flow for two different velocities. The results are even compared to an analytical model. Some specific local flow characteristics are extracted from the numerical results which give explanations about leakage backflows inside the screws and local distortion at the pump inlet section. In the same paper, an interested Non-Dimensional approach has been taken to explain the results and help the design engineer to rightly design screw pump.

In this paper, a conformal-structured moving mesh is generated for the rotor fluid domain. The mesh for stationary domains such as ports and pipes are generated by use of a commercial grid generator built into the commercial software SimericsMP $+{ }^{\circledR}$. Handling of the structured moving mesh in the CFD solver is managed by use of the user-defined function and interface [24] [25]. The main aim of this research paper is to apply novel approach for grid generation of the numerical conformal-structured moving mesh developed at City University London and to perform the full three-dimensional CFD analysis of screw pump using SimericsMP $+{ }^{\circledR}$. A cavitation model built into the commercial CFD solver SimericsMP $+{ }^{\circledR}$ has been included to correctly predict the real-time mass flow rate, rotor torque, pressure distribution, velocity field, power consumption. A standard k-epsilon turbulence model has been adopted to keep into account turbulent phenomena due to the rotation of the rotors. The results have been validated by experimental data obtained in the test rig of the Company Fluid-o-Tech.This research is part of the main's author Ph.D. program which belongs to a particular program, established by the Italian government, called PON Research \& Innovation where Italian Universities develop these Ph.D. programs in collaboration with Italian companies and foreign universities. For this specific program the other two authorities, supporting the University of Naples Federico II and the University of Sannio, are Fluid-o-Tech, located in Corsico (MI) - Italy, and the Purdue University (USA), in particular, with the Maha Fluid Power Research Center.

\section{The Twin-screw pump}

\subsection{Reference Machine and Working Principle}


A twin-screw pump is investigated; an exploded view of one flow path of the entire pump is illustrated in Fig. 1. The driving spindle consists of a double-start thread trapped $900^{\circ}$ around its corresponding shaft. The driven spindle consists of a three-start thread resulting in a 2/3 transmission ratio. The driving spindle is actively driven by the motor. Because of the helical rotation and intermeshing of the two rotors, chambers are intermittently formed at the inlet edge of the rotating screw, the liquid being continuously axially displaced from the suction chamber to the discharge end. To avoid metal-to-metal contact, several gaps exist between the stationary and the moving parts. For a given fluid (single or multiphase), these gaps and the related overall dimensions play a major role in pump performancegoverning parameters with rotational speed as well.

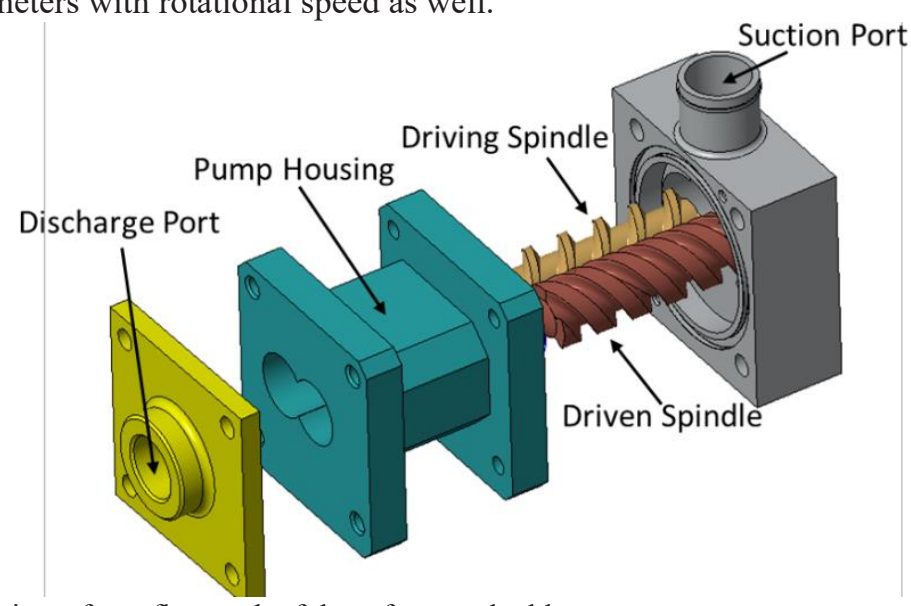

Fig. 1. Exploded view of one flow path of the reference double-screw pump.

\subsection{Gap Definition}

The intermeshing two spindles provide chambers that are encased by the spindle walls and the pump casing. The chambers are not completely sealed but connected to each other via different narrow gaps, termed circumferential, radial and flank gaps where a leakage flow occurs. The circumferential gaps are located between the spindles' tip and the casing, and the clearance between the tip of one spindle and the shaft of the other spindle corresponds to the radial gaps. Both, circumferential and radial gap locations are schematically sketched in Fig. 2. These gaps linearly move along the conveying direction. The clearances between side surfaces of spindles correspond to the flank gaps whose locations are roughly marked in Fig. 2. In contrast to radial and circumferential gaps, flank gaps exhibit a complex 3D geometry which effects a considerable difficulty regarding the set-up of a CFD model. 


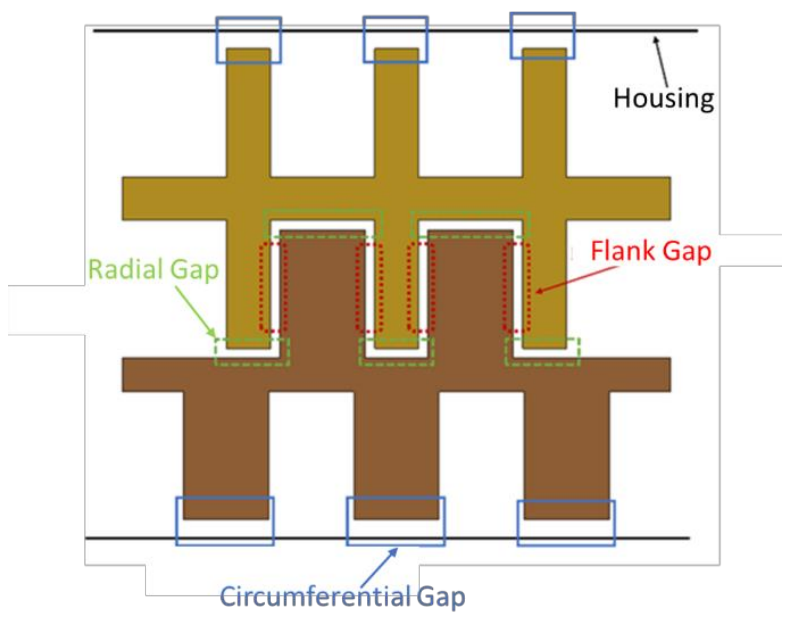

Fig. 2. A schematic view of circumferential, radial and flank gaps on a twin-screw pump with $2 / 3$ lobes arrangement.

\subsection{Test Rig}

The experiments were carried out at the Laboratory of Fluid-o-Tech, an Italian company with over 70 years of experience in the engineering and manufacturing of positive displacement pumps and fluidics system. The hydraulic schematic of the test rig in Fluid-oTech Laboratory is showed in Fig.3; the fluid is a 50:50 mixture of water and glycol (IP Antifreeze Red) at room temperature is used with a density of $1070 \mathrm{~kg} / \mathrm{m} 3$ and viscosity of $0.004 \mathrm{~Pa}$ s.

By using a control valve installed on the discharge side, a variation of operation points is achieved. Rotational speed can be continuously controlled and modified. The pressure at discharge pipe is measured by an amplified pressure transducer (Unik $5000 \mathrm{pmp}$ ). The uncertainty of pressure measurements is estimated to be lower than $0.4 \%$. The flow rate is measured in the discharge pipe by the means of a Coriolis flowmeters (Sitrans F C Mass 2100 Di 3-40) with an uncertainty below $0.15 \%$ in the interested mass flow range.

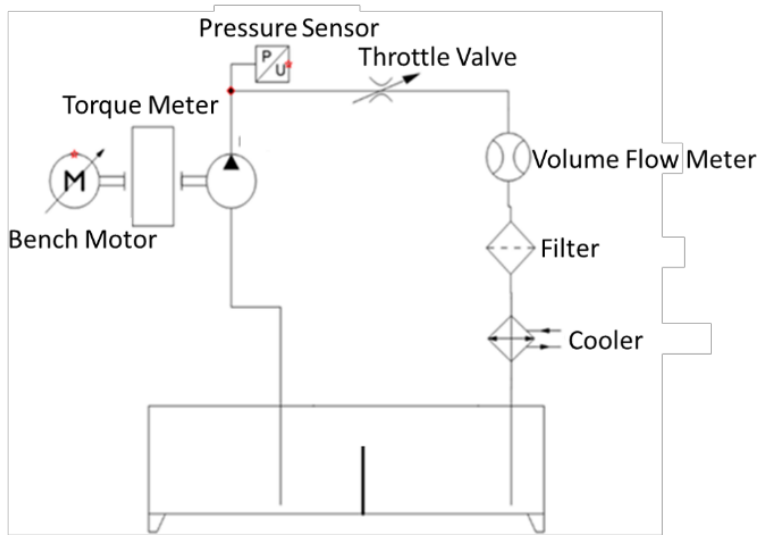

Fig. 3. Hydraulic schematic of the test rig in the Fluid-o-Tech Laboratory 


\section{Simulation Methodology}

Positive displacement screw pumps operate on the basis of changing the size and position of a working domain which consequently causes change in the pressure of the working domain thereby transporting the fluid. To calculate performance of a screw pump, quantities such as mass, momentum, energy, etc. need to be modelled. The governing equations required for the solution is a closely coupled, time dependent set of partial differential equations (PDEs) and employ a finite volume method (FVM) to be solved. The cavitating flow can be treated as a homogeneous mixture of vapour, air and liquid, in which vapour and air are gas. So, it is liquid-gas two-phase flow. Volume of fluid (VOF) considers a single effective fluid whose properties vary according to volume fraction of individual fluids. The VOF model description assumes that all immiscible fluid phases present in a control volume share velocity, pressure, and temperature fields. Therefore, the same set of basic governing equations describing momentum, mass, and energy transport as in a single-phase flow is solved. The equations are solved for an equivalent fluid whose physical properties are calculated as functions of the physical properties of its constituent phases and their volume fractions.

\subsection{Grid Generation}

Grid generation is a process of discretising a working domain of the screw pump in control volumes for which a solution of local fluid properties is to be found. The results obtained in this research work used grids generated by SCORG ${ }^{\circledR}$ (Screw Compressor Rotor Geometry Grid generator), developed at City University London. Applying the principles of analytical grid generation through transfinite interpolation with adaptive meshing, the authors have derived a general, fast and reliable algorithm for automatic numerical grid generation code. To achieve a single-domain mesh of the moving elements which is required for the CFD solver, the rotor flow domain is initially divided into two subdomains belonging to the two rotors using the rack plane. More information about grid generation could be found in Kovacevic, et al. [26] and Rane [27].

Table 2 shows the geometry parameters of rotors. An example of the numerical rack line for the working fluid domain between two rotors is shown in Fig. 4, for a reference rotor profile of an A-Type screw pump.

Table 2. Mesh configuration of fluid the domain around rotors.

\begin{tabular}{|c|c|}
\hline Circumferential division & 120 \\
\hline Radial Division & 7 \\
\hline Interlobe divisions & 100 \\
\hline Rach refinement points & 1000 \\
\hline
\end{tabular}




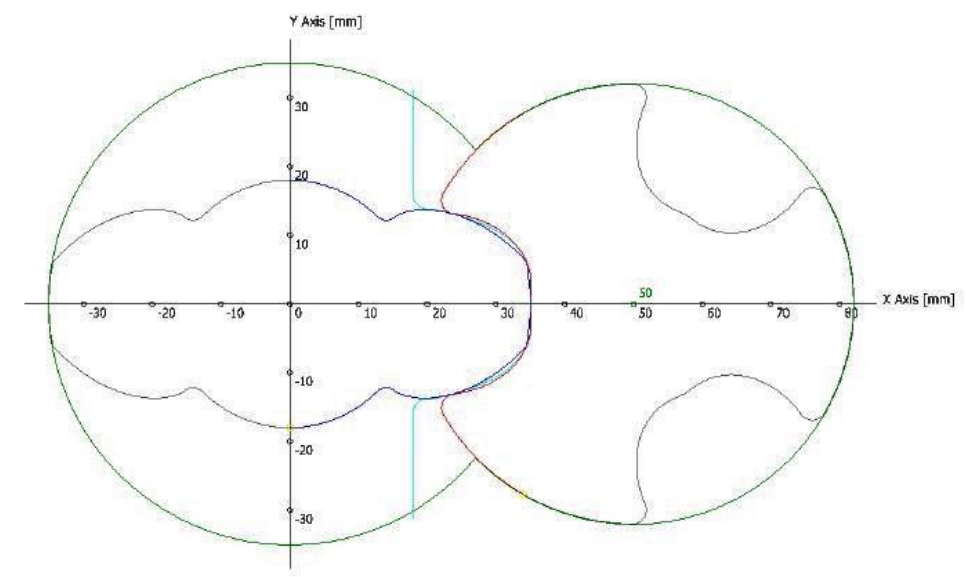

Fig. 5. Numerical rack line and typical cross section of $2 / 3$ pump profile. SCORG V5.9.0

A mesh sensitivity analysis was performed comparing simulated results with experimental data. Stable and good results have been achieved for2,3M cells with 1,3 M cells assigned to fluid domain between the rotors, while $1 \mathrm{M}$ cells related to the two ports. An unstructured body-fitted binary tree approach is used for the static mesh of the inlet port and outlet port using SimericsMP $+{ }^{\circledR}$ general grid generator; the whole fluid domain (VOF) is shown in Figure 6.

Numerical grids for rotor domains (fig. 6a) and pump port domains are connected through Mismatched Grid Interface (MGI), an implicit interface.

(a)

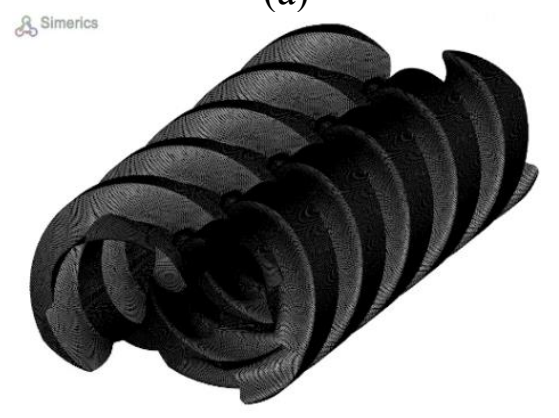

(b)

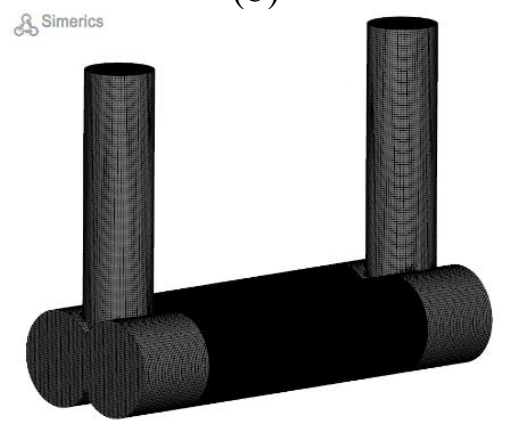

Fig. 6. Grids of the fluid domain: a) Rotor fluid domain; b) Entire fluid domain

\subsection{CFD solver}

The flow inside the double helix pump depends on the mean flow rate, the screws' relative positions versus time and the interaction between screw rotation and the pump boundary walls. The solution of the governing equations is done using the commercial software Simerics $\mathrm{MP}+{ }^{\circledR}$. Details on the solution scheme can be found in [28] and are briefly summarized here.

The numerical model is based on the Reynolds-averaged Navier-Stokes (RANS) equations with the $\mathrm{k}-\varepsilon$ model to calculate the Reynolds stresses. In Simerics MP+ ${ }^{\circledR}$, as in the original Singhal et al model [29], the working fluid in cavitating flows is always assumed to be a mixture of liquid, vapor and some non-condensable gases. By default, the cavitation 
models account for both liquid-vapor phase change and the effect of non-condensable gases. Based on the modelling approach for non-condensable gas (NGC) effect, Equilibrium Dissolved Gas Model, in which the mass fraction of the non-condensable gas dissolved in the liquid is equal to the equilibrium value, was chosen. Simulations have been run with a reference pressure of 1.01 bar and for 4 rotations of the spindles. Regarding the flow Q, time averaging is performed for one spindle revolution, after it has been assured that initial transients have been abated. The pump inlet pressure is set to $1.01 \mathrm{bar}$, and the pump outlet pressure is set at different discharge pressure up to 5.01 bar. By adjusting the pressure difference between the inlet and the outlet, the required flow rate is obtained. Rotating speed of the male rotor ranges from $500 \mathrm{rev} / \mathrm{min}$ up to $4000 \mathrm{rev} / \mathrm{min}$. Stagnation inlet and pressure outlet are used respectively for the inlet and outlet boundaries. The initial pressure and initial velocity are 1.01 bar and $0 \mathrm{~m} / \mathrm{s}$, respectively. The turbulence intensity is $1 \%$ and the turbulence viscosity ratio is 10 .

\section{Results}

The calculations were carried out with a computer powered by 8 Intel $3.5 \mathrm{GHz}$ processors and $8 \mathrm{~Gb}$ memory. Screw pump rotation was simulated by means of 60 time steps for one interlobe rotation, which was equivalent to 180 time steps for one full rotation of the male rotor, meaning in a $2^{\circ}$ main rotor rotation per step. The time step length was synchronised with each rotation speed. An error reduction of four orders of magnitude was required and achieved in 25 inner iterations at each time step. The overall performance parameters such as chamber pressure, velocity distribution, rotor torque, mass flow rate and shaft power were then calculated.

\subsection{Numerical Model Validation}

The experimental tests were carried out for several delta pressures and rotational speeds, as said, from $\mathrm{N}=500 \mathrm{rev} / \mathrm{min}$ up to $\mathrm{N}=4000 \mathrm{rev} / \mathrm{min}$. The corresponding results are given in the upper part of Figure 7 on a flow rate $(Q)$ discharge pressure $(\Delta p)$ chart while in the lower the volumetric efficiency $\left(\eta_{v}\right)$ is reported. Axes are neglected as requested by the industrial partner of the project. The agreement between experimental and simulated results is good. The maximum error is registered at the lowest rotation speed and consists of $10 \%$ of mismatch respect to the measured one. Remaining differences may be traced back to inaccuracies of the pressure loss calculation in the suction and discharge ports, due to common uncertainties by turbulence modelling and the neglecting of shaft deflection due to pressure imbalance within the spindles as will be discussed in Sec. 4.2. One further contribution might be the neglecting of the micro-motions of both spindle's axes of rotation due the unbalanced pressure field in the radial cross: in other kind of volumetric machine this has been proven to affect pump's performances, as explained by Vacca et al. [30].

In the lower part of Fig. 7 is reported the volumetric efficiency $\left(\eta_{v}\right)$ with respect to the discharge pressure. Note that it is reported only for the simulation since the comparison with the measured one is of limited use as reported by [4]: the theoretical volume can only be evaluated by the CAD model and is therefore the same for experiment and simulation as highlighted by equations (1), (2) and (3) listed below:

$$
\begin{aligned}
& Q=Q_{\text {ideal }}-Q_{\text {backflow }} \\
& Q_{\text {ideal }}=V_{\text {theoretical }} * n
\end{aligned}
$$




$$
V_{\text {theoretical }}=2 * P * A_{\text {fluid }}
$$

Where $Q_{\text {backflow }}$ represents the total mass backflow rate, as discussed in [23], $V_{\text {theoretical }}$ is the chamber volume displaced per revolution, $P$ is the pitch and $A_{\text {fluid }}$ stands for the wetted cross-sectional area of the fluid domain inside the casing and can readily be evaluated by the CAD data. Multiplication by 2 is due to double-started thread spindles.

According to Fig. 7, flow rate declines linearly with increasing the outlet pressure meaning in an increasing of back flow rate. While the $Q-\Delta p$ characteristics is essentially linear, the $\eta_{v}-\Delta p$ shows a rather declining slop towards higher speed. This is because being $V_{\text {theoretical }}$ the same, the $Q_{\text {backflow }}$ 's weight gets smaller over the speed. This sentence is in accordance with the fact that $Q_{\text {backflow }}$ is almost constant over $n$ and only depends upon $\Delta p$.

\section{Experimental results vs CFD model}
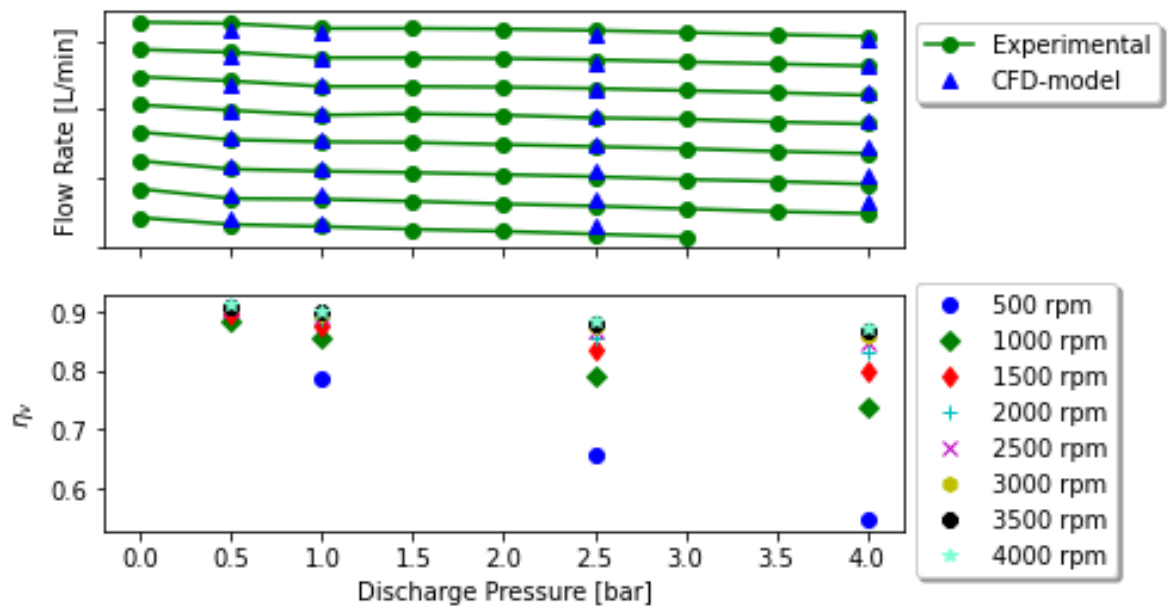

Fig. 7. Numerical model validation: comparison between experimental and simulation results in the upper part; volumetric efficiency with respect to the discharge pressure in the lower part.

\subsection{Pressure Distribution}

The 3D simulation results provide a thorough insight into the flow field within the spindles; this section is focused on the pressure contour inside the fluid domain. The pressure distribution is shown in Figure 9 both from the upper and lower view. The mean pressure gradually increases along the axial direction from the inlet to the outlet port. Due to the helical shape of the spindle, the lower portion of each chamber hurries away and thus reaches the next higher-pressure level earlier. Thus, the spindle body experiences a resulting force against the y-direction which may cause a shaft deformation. It should be noted that this deformation demands a structure simulation approach and is for the time being neglected in our simulations where we assume a perfectly rigid spindle. 


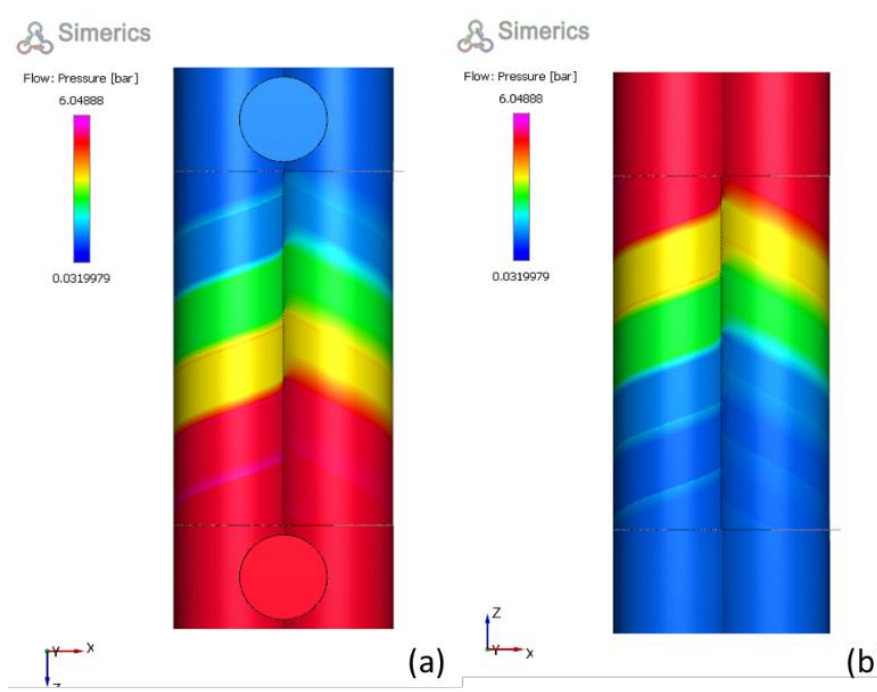

Fig. 9. Surface pressure distribution. (a) upper view; (b) lower view.

\subsection{Velocity Distribution}

Figure 10 shows the velocity contours and vectors in a specific radial cross-section of the pump. After entering the rotor intermeshing region, local velocities can reach high values related to high rotational speed. Some negative axial velocity regions can be observed, directing towards the inlet pipe section, located close to the gap between the helix addendum circle and the lining inner bores, that corresponds to the main leakage areas. High velocity distortions also exist at the exit end of the pump. The presence of a higher pressure difference over the flank gap has been discussed already in Sec. 4.1 and leads to a higher velocity and consequently larger backflow rate inside the flank gap compared to the circumferential gap. Thus, this is the first study that figures out that also the flank gap may be important.

In each circumferential gap, a step pressure rise occurs in flow direction which is from the opposite point of view associated with a pressure drop in gap backflow direction. A thorough inspection of the 3D flow field reveals that the magnitude of the circumferential velocity in the lower portion of any chamber is higher than in the upper one which is due to the fact that the spindles are counter-rotation. This higher circumferential velocity not only causes higher pressure losses in the lower portion of the chamber which consequently leads to a reduction of total pressure, but also increases the dynamic pressure. The reduction of total pressure on the one hand and the rise of the dynamic pressure on the other hand leads to lower static pressure at the bottom part of any chamber. Therefore, a locally negative pressure level may serve as an indicator of a cavitation-flow insurgence. Cavitation may occur at the spindle leading edge, the radial gaps and the flank gaps, as show in Fig. 11. It should be pointed out that with a speed decrease, the extend and level of negative pressure decline (not shown here) so that it can be clearly concluded that the occurrence of cavitation is more pronounced with higher speed. 


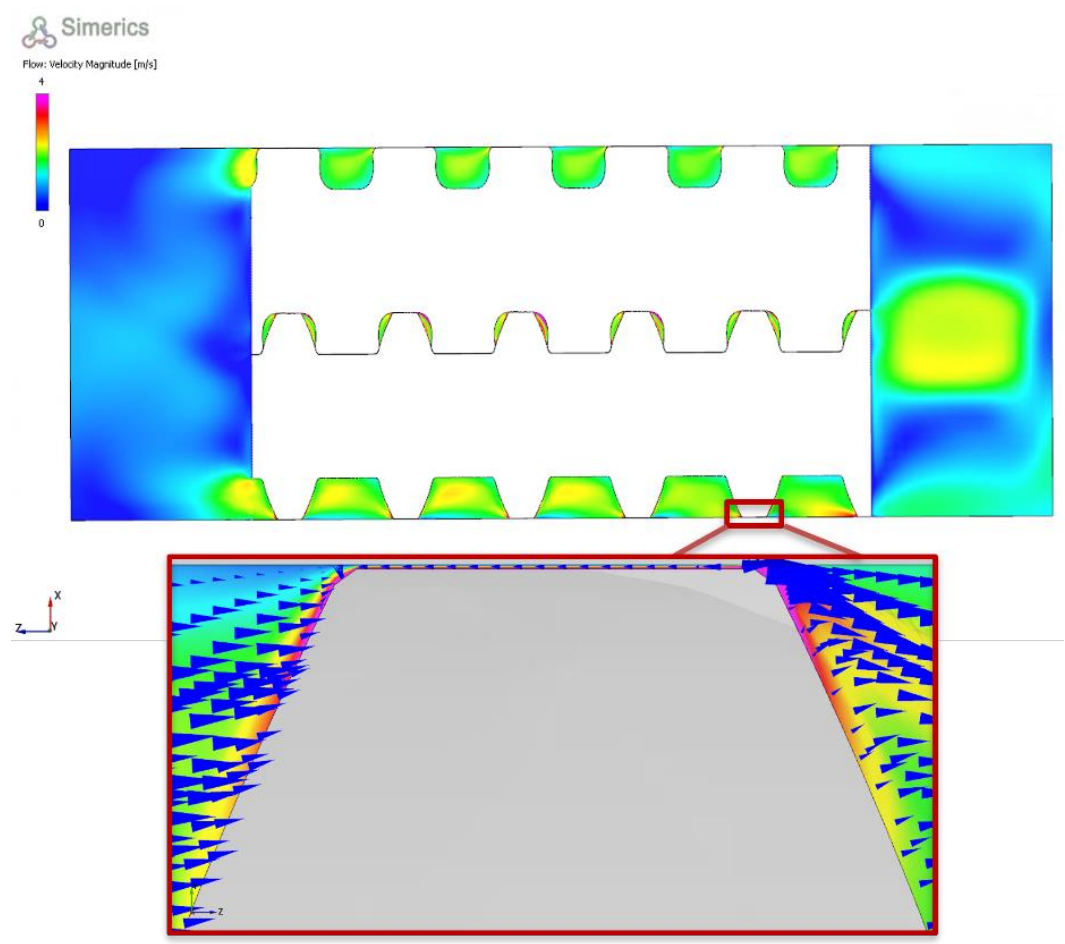

Fig. 10. Local velocity distribution. Surface contour and vectors of a y-cross section.

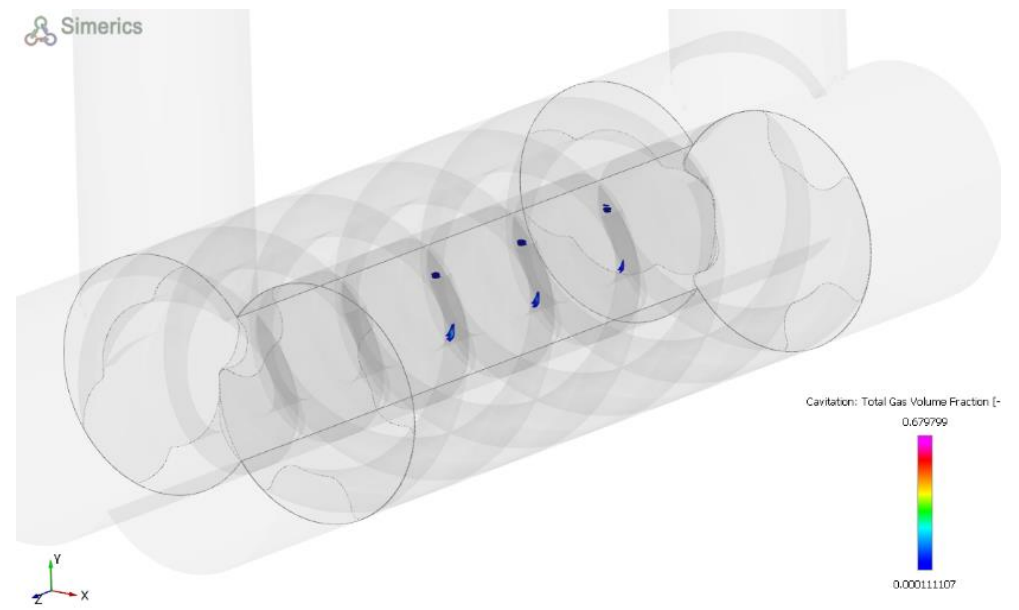

Fig. 11. Isosurface of the total gas volume fraction at $3000 \mathrm{rev} / \mathrm{min}$ and $\Delta \mathrm{p}=1$

\section{Conclusions}

A full 3D CFD simulation of twin-screw pump has been carried out using structured moving numerical mesh through the coupled using of SCORG ${ }^{\circledR}$ and Simerics MP+ ${ }^{\circledR}$. The calculation results were validated with experimental data confirming the accuracy and 
applicability of the proposed numerical method for performance prediction of screw pumps. Overall performance measurements were performed using a 50:50 mixture of water and glycol as the working fluid. As expected, the leakage amount was found to be independent of the rotational speed and only depends on the pressure difference.

The screw pressure gradually increases from the inlet end face along the axial direction of the screw to the outlet end face, and the pressure near the screw inlet end face is negative. The pressure distribution in the screw groove is relatively uniform, but the pressure distributions in the screw clearance and the meshing area are different from that in the screw groove. The relative leakage flow reduces with the increase in rotational speed. It increases with the increase in the discharge pressure. The difference between the measured and calculated mass flow rates decreases with the increase in speed.

The cavitation intensity and distribution area were predicted based on the Singhal model [27]. If it appears, the cavitation will mainly happen in the area close to the suction port and will be distributed in the outward surfaces of rotors and clearances. The increase of rotation speed will enlarge the intensity of cavitation observably in screw pump. The increase of the discharge pressure at constant speed of rotation will increase the amplitude of volume fraction, while the average of volume fraction nearly remains the same.

The study of use of CFD for screw pumps provides a better understanding of the internal flow field characteristics and provides a good basis for the next-step research on multiphase flow and cavitation in screw pumps.

\section{Acknowledgment}

The authors would like to thank the support from Pierpaolo Lucchesi and Giuseppe Ricucci of Fluido-Tech, from Federico Monterosso and Micaela Olivetti of OMIQ s.r.l. and SCORG ${ }^{\circledR}$ for providing the software.

\section{References}

[1] Li F. Screw pump [M]. Beijing, China: Machine Press, 2010.

[2] Nie S, Li F, Lv W, et al., 2009, Improvement and hermeticity analysis of B-type double screw pump curve. Mech Electric Equip, 26, pp.47-51.

[3] Vetter, G., and Wincek, M., 1993, "Performance Prediction of Twin-Screw Pumps for Two- Phase Gas/Liquid Flow," Pumping Machinery, Fluid Engineering Conference (FED), ASME, Washington, DC, 154, pp. 331-340.

[4] Vetter, G., Wirth, W., Korner, H., and Pregler., 2000, "Multiphase Pumping with TwinScrew Pumps- Understand and Model Hydrodynamics and Hydroabrasive Wear," $7^{\text {th }}$ International Pump User Symposium, pp.153-169.

[5] Mewes D, Aleksieva G, Scharf A, et al., 2008, Modelling twin screw multiphase pumps - a realistic approach to determine the entire performance behaviour. In: $2^{\text {nd }}$ International EMBT Conference, Hannover, April 2008, pp.104-116.

[6] Patil A. Performance evaluation and CFD simulation of multiphase twin-screw pumps. PhD Thesis, Texas A\&M University, College Station, TX, 2013.

[7] Chan E. Wet-gas compression in twin-screw multiphase pumps. MS Thesis, Texas A\&M University, College Station, TX, 2006.

[8] Rabiger K. Fluid dynamic and thermodynamic behavior of multiphase screw pumps handling gas-liquid mixtures with very high gas volume fractions. PhD Thesis, Faculty of Advanced Technology, University of Glamorgan, 2009.

[9] Rabiger K, Maksoud T, Ward J, et al., 2008, Theoretical and experimental analysis of a multiphase screw pump, handling gas-liquid mixtures with very high gas volume fractions. Exp Therm Fluid Sci, 32, pp. 1694-1701. 
[10] Cao F, Peng Y, Xing Z, et al., 2001, Thermodynamic performance simulation of a twinscrew multiphase pump. Proc IMechE. Part E: J Process Mechanical Engineering, 215, pp. $157-163$.

[11] Hu B., Cao F., Yang X. Theoretical and experimental study on conveying behavior of a twin-screw multiphase pump [J]. Proceedings of the Institution of Mechanical Engineers, 2016, 230(4), pp. 304-315.

[12] Liu P., Patil A., Morrison G., 2018, Multiphase flow performance prediction model for twin-screw pump [J]. ASME Journal of Fluids Engineering, 140(3), 031103.

[13] R. Ma and K. Wang, 2009, CFD Numerical Simulation and Experimental Study of Effects of Screw-Sleeve Fitting Clearance upon Triangular Thread Labyrinth Screw Pump (LSP) Performance. Journal of Applied Fluid Mechanics, Vol. 3, No. 1, pp. 75-81, 2010.

[14] Tang Q., Zhang Y., 2014, Screw optimization for performance enhancement of a twinscrew pump [J]. Proceedings of the Institution of Mechanical Engineers, 228(1), pp.73-84.

[15] Tang Q and Zhang Y., 2014, Screw optimization for performance enhancement of a twin-screw pump. Proc IMechE. Part E: J Process Mechanical Engineering, 228, pp.73-84.

[16] Qian Tang, Yuanxun Zhang, Linqing Pei, Jiong Tang, 2011, Optimal design of screw and flow field analysis for twin-screw pump, Proceedings of the ASME 2011 International Design Engineering Technical Conferences \& Computers and Information in Engineering Conference IDETC/CIE 2011 August 28-31, 2011, Washington, DC, USA

[17] Kovacevic' A, Stosic' N and Smith IK. Screw compressors: three-dimensional computational fluid dynamics and solid fluid interaction. New York, NY: Springer.

[18] Stosic N, Smith IK and Kovacevic A. Screw compressors: mathematical modelling and performance calculation. New York, NY: Springer, 2005.

[19] Yan D., Kovacevic A., Tang Q. Numerical modelling of twin-screw pumps based on computational fluid dynamics [J]. Proceedings of the Institution of Mechanical Engineers, 2017, 231(24), pp. 4617-4634.

[20] Yan D., Tang Q., Kovacevic A. et al. Rotor profile design and numerical analysis of 23 type multiphase twin-screw pumps [J]. Proceedings of the Institution of Mechanical Engineers, Part E: Journal of Process Mechanical Engineering, 2018, 232(2): 186-202.

[21] Hai-Tao YU, Qi-Fei YU, Bo LI, Chang-Lin QIU and Guo-Xiang HOU, (2017), Numerical Simulation of Flow in Symmetry Twin-screw Pump based on PUMPLINX.

[22] Di Zhang, Li Cheng, Ying-yuan Li, Wei-xuan Jiao, 2020, The hydraulic performance of twin screw pump, Journal of Hydrodynamics, 32(3), pp. 605-615

[23] W. Zhang, Q. Jiang, G. Bois, H. Li, X. Liu, S. Yuan, Y. Heng, 2019, Experimental and Numerical Analysis on Flow Characteristics in a Double Helix Screw Pump - Energies, 12, 3420 .

[24] Corvaglia, A.; Rundo, M.; Casoli, P.; Lettini, A. Evaluation of Tooth Space Pressure and Incomplete Filling in External Gear Pumps by Means of Three-Dimensional CFD Simulations. Energies 2021, 14, 342. hiips://doi.org/10.3390/en14020342

[25] Corvaglia A, Ferrari A, Rundo M, Vento O. Three-dimensional model of an external gear pump with an experimental evaluation of the flow ripple. Proceedings of the Institution of Mechanical Engineers, Part C: Journal of Mechanical Engineering Science. 2021; 235(6):1097-1105. doi:10.1177/0954406220937043

[26] Kovacevic A, Stosic' N and Smith IK. Screw compressors three-dimensional computational fluid dynamics and solid fluid interaction. Berlin: Springer, 2006.

[27] Rane S. Grid generation and CFD analysis of variable geometry screw machines. $\mathrm{PhD}$ Thesis, City University of London, London, 2015.

[28] Inc. S. Simerics MP+'s User Manual - v 5.0.11.

[29] A. Singhal, M. Athavale, H. Li and Y. Jiang, 2002, Mathematical basis and validation of the full cavitation model, J. Fluids Eng., 124, pp. 617-624 
[30] Vacca A., Guidetti M., Modelling and Experimental Validation of External Spur Gear Machines for Fluid Power Applications, Simulation Modelling Practice and Theory, Vol. 19, No.9, pp.2007-2031, 2011. 